Article

\title{
The Post-Intervention Persistence of Energy Conservation Behaviors: An Evaluation of the 'Start Green' Program
}

\author{
S. Barnett Burns ${ }^{1,2}$ and Beth Savan ${ }^{1, *}$ \\ 1 School of Environment, University of Toronto, Toronto, ON M5S 3E8, Canada; \\ s.barnett.burns@mail.utoronto.ca \\ 2 Integrated Program in Neuroscience, McGill University, Montreal, QC H3A 0G4, Canada \\ * Correspondence: b.savan@utoronto.ca
}

Received: 12 December 2017; Accepted: 8 March 2018; Published: 14 March 2018

\begin{abstract}
For more than four decades, behavioral intervention programs informed by psychology have been employed to increase pro-environmental behaviors. However, there has been little evidence for the post-intervention durability of target behaviors. The few studies that have conducted such evaluations have found that improvements often return to baseline levels post-intervention. This study evaluated the durability of home energy conservation behaviors before, during, and after a community based multi-technique intervention program, and examined the relationship between behavioral durability and the perceived importance, convenience and family norms of each behavior, as well as generalized pro-conservation decision making. The results show increased frequency in target behaviors that remain elevated seven months post-intervention. While the reported generalization of pro-conservation decision-making consistently increased during the study, perceived importance, convenience, and family norms of target conservation behaviors were largely unaffected. In addition, the few significant alterations in these perceptions were found to be due to increases during the post-intervention period only, indicating that they are not necessary pre-requisites for durable behavior change. These results show that a well designed community based intervention can have direct impacts on target behaviors that persist beyond its termination.
\end{abstract}

Keywords: conservation behavior; pro-environmental behavior; behavioral intervention; field intervention; durability; persistence; social norms; energy

\section{Introduction}

Since the early 70's there has been a growing awareness of the significant role human behaviors play in many pressing environmental issues. In an attempt to increase a wide variety of pro-environmental behaviors, behavioral intervention techniques derived from social and behavioral psychology have been employed as part of public intervention programs. Evaluations of the varying successes of these interventions have suggested that the strategic use of many techniques, such as information, commitment, prompts, feedback, incentives, role models, and social reinforcement, can influence individual habits and choices resulting in the short-term reduction of resource consumption [1-6]. However, these evaluations often limit their focus to standard evaluative measures such as significance of effect, reliability, effect size, and cost-effectiveness. This may not be sufficient to determine the long-term success of programs, nor the overall effectiveness of large-scale field applications [1,3,6]. De Young [3], suggested that in addition to the more common assessments, techniques should also be evaluated based on speed of change, particularism, or how specifically a technique appeals to individuals based on their group membership, and durability. Cone and Hayes [7] have argued that interventions should be assessed in terms of how generally the technique can be 
applied across environmental behavioral domains, circumstances and contexts. Yet other researchers recommend the examination of qualitative narratives to capture the meaning/motivations behind behavior change and lived experience [8-10]. One of the most important omissions has been an evaluation of the long-term post-intervention impact of interventions. Indeed, the few studies that have looked at long-term post-intervention behavior have suggested that many behaviors return to baseline after the intervention is discontinued [1]. This potential for post-intervention reversal is clearly problematic and impacts many other measures as well. For example, an intervention program quickly loses its cost-effectiveness if it has to be re-employed multiple times to maintain a desired behavior. Thus, the only reliable way to predict the long-term success of a program is to evaluate changes in behavior beyond the termination of the intervention. Many reviewers agree that evaluating the long-term durability of behavior change is important $[1,3-6,11,12]$. Indeed, if pro-environmental behaviors revert to baseline as soon as a program is discontinued it cannot be considered an effective intervention.

\subsection{Behavior Durability}

An obvious way to create persistence is potentially through the use of permanent interventions. Both continuous feedback devices and changing defaults to conservation settings on appliances have the potential to be very cost-effective [13]. There have been positive effects in cases where continuous feedback devices have been installed in smart meters [14], showers [15] and appliances [16]. Although these studies are limited in their duration, these positive effects may have more chance of persisting because the intervention is never discontinued. However, more follow-up studies are needed as the effects seen may be attributable to the novelty of the new device or a pre-existing goal to reduce energy use, and thus may not persist in all situations [14,16]. In addition, despite continuous real time feedback being effective in most studies, Delmas et al. [2] concluded in their meta-analysis that even continuous feedback is not sufficient on its own to produce persistent energy conservation behaviors.

The durability of more temporary interventions has not received much research attention, most likely due to the fact that it is methodologically difficult and because questions of behavioral persistence are complicated. For example, many of the techniques that are highly successful in the short-term are not as successful over the long-term and visa-versa. Incentives, for example, usually produce significant effects very quickly, however, the few studies that have done post-intervention analysis have found that as soon as the incentives are removed, participants return to baseline behaviors $[11,17,18]$. Techniques that have shown the most promise for creating persistent pro-environmental behavior tend to work more slowly. Techniques that create an internal change such as commitment, feedback (combined with personal goal setting and monitoring), direct experience, intrinsic motivation, and Community-based research (CBR) programs that create a strong sense of collective identity by altering social norms and values, tend to have smaller initial effects, but also tend to create self-maintaining behaviors over time $[3,19,20]$. The few studies that have found evidence of durable behavior changes, even after interventions have been discontinued, have looked at interventions that were using techniques like commitment and feedback combined with goal setting $[11,17,18,21-25]$. These techniques combined with a social element, such as peer feedback, public commitment, social marketing pressure, or community-based participation, appear to be even more promising in their ability to create persistent behaviors [21,22,24,26-29], and are popular in public health initiatives for that reason [30]. As is suggested by many social psychology theories such as social identity pathway theory [31,32], perceived personal control [33], citizen participation [34], self-efficacy [35] and empowerment [36], the self-determined participatory element of these highlighted techniques, along with the engagement in communal action, may lead to a new self-definition that includes a sense of belonging, ownership, and responsibility to contribute to the collective group and act as a change agent. 


\subsection{Summary and Research Questions}

Although the significance of effect, reliability, effect size and cost-effectiveness are important measures when evaluating pro-environmental behavioral intervention programs, it is not enough to stop there. Evaluating interventions while the program is still running tends to give at best an incomplete, and at worst an artificial picture of the effectiveness of the program. How participants behave during an intervention does not necessarily reflect how they will behave once the intervention is discontinued, therefore any long-term conclusions based on these evaluations are likely to be erroneous. Thus, the durability of behavioral changes after an intervention has been discontinued is not only an important criterion to measure, but also gives a more complete picture of other criteria, such as cost-effectiveness and the overall impact of a program.

This paper evaluates the Start Green intervention program, which was implemented at the University of Toronto between September 2010 and February 2011. Like most intervention programs, Start Green was limited in its reach and duration, however it also integrated many of the CBR techniques that have been previously associated with durable changes. By measuring energy conservation behaviors, and the perceived importance, family expectations, and ease of each behavior as well as generalized conservation decision making before, during, and seven months post-intervention, this paper not only shows a rare example of the potential of intervention programs to create long-term increases in conservation behaviors that persist beyond the program, but also challenges the assumption that increased attitudes of conservation importance, ease, and social norms are necessary pre-requisites to durable behavior changes.

\section{Materials and Methods}

'Start Green' is a behavioral intervention program targeting energy conservation behaviors in the home. It is an off-campus version of the "REWIRE" program, which utilizes Community Based Social Marketing [12,20], and other types of CBR techniques [30] to encourage increased energy conservation in campus student residences [37]. REWIRE is a modular intervention program developed by a team at the University of Toronto Sustainability Office and has been successfully implemented at campus student residences at the University of Toronto [37,38].

\subsection{Intervention Design}

\subsubsection{REWIRE Design}

Each module in REWIRE is a complete set of social psychology and community organization techniques that synergistically target a specific behavior (e.g., using cold water to wash clothes). Modules are created by first identifying the unique barriers to the desired behavior that exist in a specific population, and subsequently identifying the applicable techniques or training to overcome these barriers. Intervention techniques usually include commitments, prompts, social norms, feedback, motivation building, personalized/tailored communication or information, and limited incentives and disincentives. Modules are tailored both to the target behavior and to the target participants and utilize the existing community structures and social norms.

\subsubsection{Start Green Modules}

Start Green consisted of six REWIRE type modules which were implemented monthly, starting in September 2010 in the following order: participant commitment, cooling awareness, lighting and electricity awareness, heating awareness, laundry and water awareness, and elevator use awareness (see Table 1). The modules consisted of tailored information about the target behavior, posters, stickers and other prompts, as well as a green-house gas (GHG) reduction kit containing relevant tools to reduce barriers. The modules were designed in collaboration with the program participants via community meetings, focus groups, interviews, and surveys and were also informed by past iterations of REWIRE. This collaboration included an analysis of the unique barriers residents faced to conserve energy and 
the recruitment of student volunteers to represent a few floors in their own buildings in order to keep in contact with neighbors, encourage participation, install and use kit contents, plan activities and events, post and distribute prompts, and gather feedback from participants throughout the intervention.

Table 1. Description of behavioral modules and supplies provided during the intervention program from September 2010 to February 2011.

\begin{tabular}{|c|c|c|c|}
\hline Month & Module & Target Behaviors & Kit Supplies \\
\hline September & Commitment & Make a public commitment to increase energy conservation & Commitment poster \\
\hline October & Cooling & $\begin{array}{l}\text { Reducing AC use } \\
\text { Increasing fan use } \\
\text { Increasing window shading-closing drapes during the daytime } \\
\text { Opening windows to circulate air }\end{array}$ & \\
\hline November & Lighting and electricity & $\begin{array}{l}\text { Reducing the use of lights and electronics when not in use } \\
\text { Using CFL bulbs }\end{array}$ & 2 CFL bulbs \\
\hline December & Heating & $\begin{array}{l}\text { Increasing insulation of windows-close drapes at night } \\
\text { Increasing space heating } \\
\text { Reducing temperature } \\
\text { Wearing appropriate warm clothing }\end{array}$ & Window insulation film \\
\hline January & Water and laundry & $\begin{array}{l}\text { Reducing hot water use } \\
\text { Increasing cold water washing cycles } \\
\text { Reducing shower length } \\
\text { Reducing the running of taps } \\
\text { Reducing dryer use }\end{array}$ & $\begin{array}{l}\text { One low-flow showerhead } \\
\text { Cold water laundry detergent } \\
\text { Laundry drying rack }\end{array}$ \\
\hline February & Elevator & $\begin{array}{l}\text { Reducing elevator use } \\
\text { Increasing stair use as a health promotion }\end{array}$ & \\
\hline
\end{tabular}

\subsection{Setting}

The Start Green program was implemented in an off-campus student residence known as Student Family Housing (SFH), which consists of two high-rise apartment buildings near the University with a total of 35 floors and approximately 700 private units. In comparison to REWIRE the SFH buildings have reduced communal space. The apartments in SFH are private with their own kitchen and bathroom facilities. However, there are common hallways, main entrances and community and social rooms in the buildings, which were used for regular communication via posters and notices and community events. The buildings have elevators, common laundry rooms, a central hot water heating system, and no central air, but many window AC units. Since the buildings are located in Toronto, Ontario, Canada, the main sources of energy at the time of the intervention were nuclear, hydroelectric, coal and natural gas. A thorough vetting of the utilities at student family housing buildings was conducted via walkthroughs and meetings with the building management to identify how much control residents have over their energy use. Residents in each apartment had control over their laundry, air conditioning, electricity use, and heating thermostat, however electricity usage was metered centrally. All utility costs were included in the rent, therefore the residents did not receive monthly bills or information on energy use. Due to the old infrastructure in the buildings it was not possible to separately monitor energy usage in each unit. In addition both buildings were undergoing a multi-year process of improving the infrastructure and installing energy efficient upgrades. This made it impossible to separate out, and accurately attribute, quantifiable energy savings. Because of this, energy use data was not included in the evaluation of the Start Green program.

\subsection{Participants}

Student Family Housing households consist of at least one University of Toronto student and his or her partner and/or children. While REWIRE focused on first and second year undergraduate students, the participants of Start Green encompassed a much wider demographic range. The majority of the student residents ( $70 \%$ of survey respondents) were graduate students at the University of Toronto who receive a guaranteed minimum stipend as long as they are enrolled in their program. As it is a pre-requisite to living in the building, student residents either lived with a spouse and/or children. $60 \%$ of the student residents were international students and came from 60 different countries. 
At least $43 \%$ of the 700 apartment units, or 301 student residents, made a public commitment to the program at baseline, and $47 \%$, or 329 residents, responded to at least one survey. Although there was significant overlap between the survey respondents and the public commitment participants, it is impossible to know the exact extent of overlap as only $56 \%$ of the survey respondents provided their unit numbers on the surveys, which was the only identifiable information we collected. Although it may appear strange that more student residents responded to surveys then made public commitments to the program, there were multiple other Start Green events, as detailed below, that residents could participate in, such as peer mentoring interactions, greenhouse gas reduction parties, and swap events for example. Student Family Housing building management kept track of the email addresses of student residents who participated, and administered the surveys. The surveys were only emailed to student residents who had participated in the program. Student residents who moved in during the intervention or during the follow-up period were not sent surveys. Although we only targeted the student residents with the surveys, many non-student spouses/partners or children were also active participants in the common actions and social events and could have been involved in answering the surveys, thus the total reach of the program was much larger. We estimate that the total reach of the program was over 600 adults and children, as all building residents were exposed to the prompts and tailored information, they may have been influenced by the program, and in turn, may have influenced the target student residents.

\subsection{Intervention Techniques}

\subsubsection{Peer Mentoring and Workshops}

Student volunteer representatives (SVRs) were recruited during the initial barrier analysis to represent and help implement the program in both buildings. There were approximately 5-6 student representatives in each building for a total of 10-12 during any given module. These individuals represented their own floor and often a few floors above and below them in their building. They were given Start Green t-shirts, scarves and buttons and trained in multiple workshop sessions on how to interact with their neighbors to encourage pro-environmental behaviors. Training workshops were offered during the modules and consisted of information about the target behaviors and their importance for reducing energy, use of the supplies provided in the kit, as well as event planning exercises, and a volunteer information booklet. The techniques that were taught ranged from how to ask their neighbors about energy conservation efforts during casual conversations to how to plan GHG reduction parties and arrange more formal meetings or training sessions when participants were having trouble using the provided supplies or module instructions in their homes. The representatives were responsible for organizing the giveaways for each module, which gave them the opportunity to meet their neighbors and encourage them to co-operate in helping make their building more environmentally friendly. They also integrated Start Green messages into other social events in the buildings.

\subsubsection{Other Social Norms}

GHG reduction parties with at least 4 residents were organized by SVRs in their units in order to familiarize their neighbors with the GHG reduction kit contents, demonstrate installation and use, and create opportunities for peer-learning on the behavioral aspects of using the energy saving devices. Over 300 of these parties were organized and a total of 220 GHG kits were given out. Therefore the GHG parties reached an estimated 800 residents (including those attending parties and family members of those receiving kits). The parties were held in each building at the beginning of each module to generate social interaction and excitement about the program. T-shirts and scarves with Start Green branding were printed and given out during these parties to create a sense of belonging and communal effort. As mentioned above, the kits were distributed at these events and public commitment pledges were taken from residents. In addition, two "swap events" were organized in November 2010 and 
February 2011 to foster community participation, form strong social ties, and further promote a social norm of energy conservation (Figure 1). The Start Green organizers and the student representatives organized a donation period over a week for each building and, at the end of this week, hosted a day-long swap event where residents could trade their belongings for their neighbors' belongings. 144 residents participated in the swap events. Any leftover items were donated to charities. The items that were gathered, exchanged and/or donated were recorded and analyzed to assess the green-house gas emission reductions from landfill diversion.

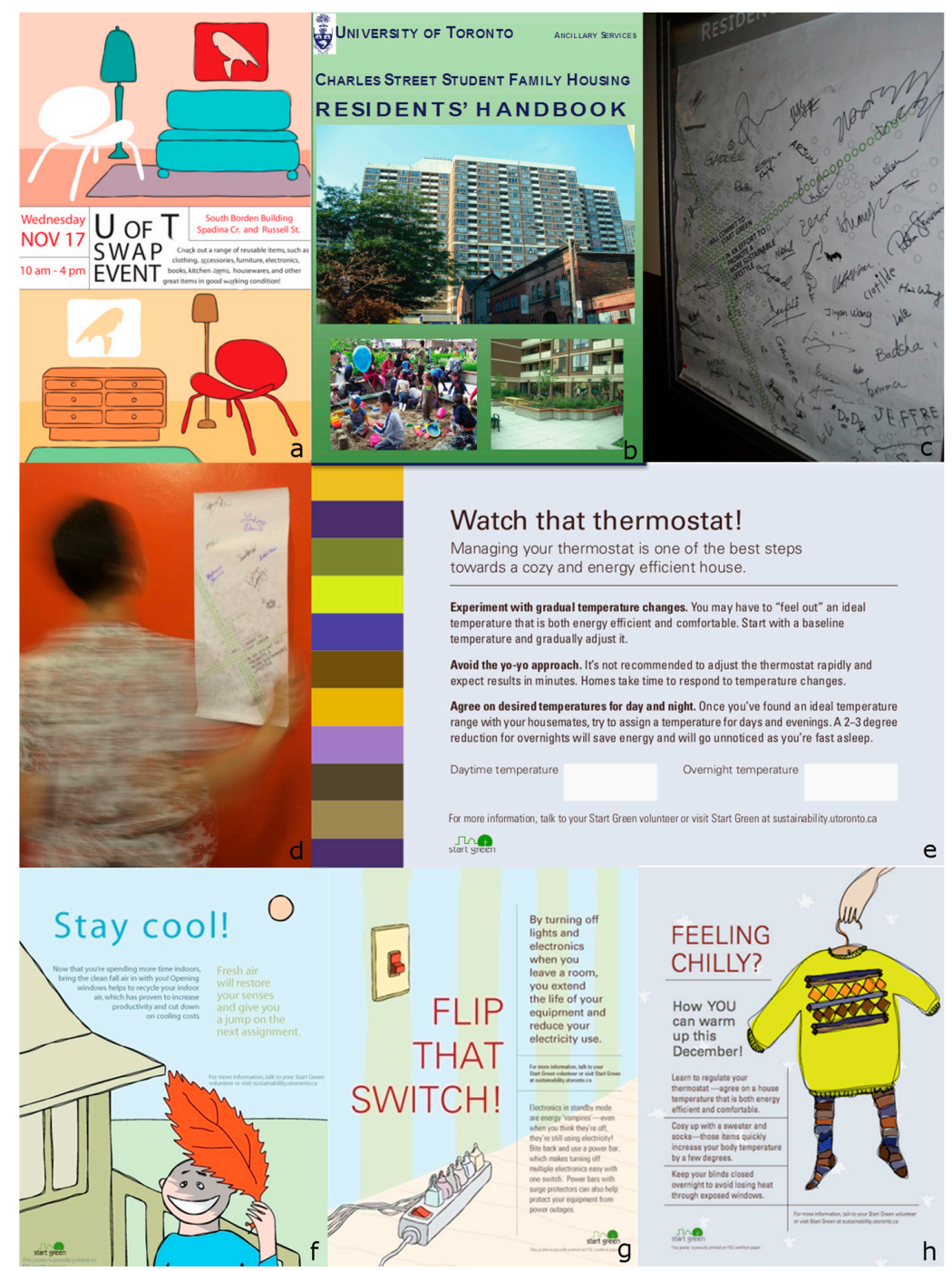

Figure 1. From top left to bottom right: (a) swap poster; (b) residents handbook; (c,d) commitment posters; (e) heating module commitment; (f) cooling module; (g) lighting and electricity module; (h) heating module.

\subsubsection{Commitments}

Residents were asked to make a public written pledge to reduce their energy consumption and participate in the program during the launch month of Start Green. They signed a public poster that was fixed in a communal space (Figure 1). Student volunteers recorded and tracked commitments made by residents on their floors. Residents also signed commitment pledges in public when they received kit supplies during each module kit distribution event (Figure 1). They pledged to use, install and/or share the items they received in an effort to conserve energy and reduce green-house gas emissions. 


\subsubsection{Prompts}

At the beginning of each module large posters and small stickers detailing the target behaviors were posted in multiple highly visible common areas, such as hallways and entrance lobbies (Figure 1). In addition, prompts were placed in key behavioral zones such as stickers on washing machines to encourage using cold water washes.

\subsubsection{Tailored Information}

Participants received booklets on the infrastructure of their buildings detailing the areas in which energy conservation was important and possible and what would make the most impact (Figure 1). They received instructions and suggestions on how to perform the module target behaviors using the existing building infrastructure and the supplied kits. An email was also sent to each student resident at the beginning of each module and a page was set up on the Sustainability Office website with additional information.

\subsubsection{Barrier Reduction Materials}

Participants were given GHG reduction kits as part of the GHG reduction parties as mentioned in the module design section above. These materials consisted of useful items that would enable residents to begin to reduce energy, such as compact fluorescent light bulbs. These were not given as a reward but supplied in order to decrease barriers to adoption of target behaviors.

\subsection{Surveys}

Four online surveys were administered to the residents. The first survey was delivered to all the units and answered by $7 \%$ of the student residents. The purpose of this survey was to identify the unique barriers that were preventing residents from engaging in energy conservation behaviors. This information was used in combination with information from community meetings, focus groups, and interviews with staff to inform the design of each intervention module and decide on the GHG kit contents. The second (baseline), third (closing) and fourth (follow-up) surveys were identical to each other and were used to assess the performance frequency of specific conservation behaviors, as well as perceived behavioral importance, family social norms, behavioral ease, and generalized conservation decision making. The baseline survey was distributed to all the units in September 2010, before the commencement of the program to assess control levels of behavior, attitudes, values, social norms and habits, and was answered by 59 student residents. The closing survey was distributed to all the units and was answered by 221 student residents. The follow-up survey was distributed seven months after the termination of the program in September 2011 to the student residents who had responded to the third survey, with 64 respondents. This last survey was limited to participants who had previously answered the third survey in order to exclude residents who had not been exposed to any aspect of the program. During the seven-month post-intervention period there was a degree of turn-over in the building. Residents who had recently moved in, or who had otherwise not participated in the Start Green program at all were identified by the building management team and were not sent the survey. For confidentiality issues, the Start Green programmers and researchers did not have access to this information. The baseline, closing, and follow-up surveys asked an identical set of questions that can be separated into five categories: behavioral, perceived behavioral importance, perceived family norms, perceived behavioral convenience, and generalized conservation decision making.

\subsubsection{Behavioral Questions}

The first nine questions directly asked how often the participant performed a specific pro-environmental behavior assessed on a 5-point Likert scale from 1 (never) to 5 (always). For example: Please indicate how often you do the following: I turn off lights when I leave rooms and hallways in 
my apartment. These questions averaged at the 3 time points were used to determine the persistence of post-intervention behavioral change.

\subsubsection{Perceived Behavioral Importance Questions}

This set of nine questions queried how important the target behavior was to the participant on a 5-point Likert scale from 1 (strongly disagree) to 5 (strongly agree). This perceived importance is assumed to reflect the attitude of the participant towards a specific pro-environmental behavior. For example: Please indicate your level of agreement with the following: It is important to turn off lights in rooms and hallways when I am not using them.

\subsubsection{Perceived Family Norm Questions}

These nine questions assessed how strongly the participant felt that there was an expectation in their family culture to perform specific behaviors, assessed on a 5-point Likert scale from 1 (strongly disagree) to 5 (strongly agree). For example: Please indicate your level of agreement with the following: My family members expect me to turn off lights in rooms and hallways when I am not using them. These questions and how they changed over the course of the intervention gave an indication of how the program impacted family members and therefore gave an indication of the proliferation beyond the target individuals.

\subsubsection{Perceived Behavioral Convenience Questions}

These nine questions assessed the perceived convenience of a particular behavior or action. Since perceived convenience tends to increase as habits increase, these questions can be seen as a measure of how internalized and habitual the behavior has become. For example: I find it easy to turn off lights in rooms and hallways when I am not using them, assessed on a 5-point Likert scale from 1 (strongly disagree) to 5 (strongly agree).

\subsubsection{Generalized Conservation Decision Making}

A single question assessed how strongly the participant had generalized their pro-environmental behaviors to their life and thus may give a measure of how strongly they have identified with being a pro-environmental individual and how likely they were to apply this in other areas of their life. This question therefore gave an indication of the potential for proliferation of conservation behaviors beyond the target behavioral domains: How often do you make decisions with the environment in mind, assessed on a 5-point Likert scale from 1 (never) to 5 (always).

\subsection{Data Analysis}

In this paper, we compare mean ranks of self-reported behaviors, attitudes, social norms, habits and values at baseline, closing and follow-up time points to evaluate the intervention and post-intervention effects of the Start Green intervention program. We also assess if alterations in energy conservation behavior durability co-vary with alterations in perceived importance, convenience, and family norms of those behaviors, as well as the overall generalization of conservation decision making.

It would have been preferable to analyze the three surveys in a repeated measures design to increase power. However, part of the CBR approach is to foster trust in the community in order to maximize participation, increase retention and thus ensure a more valid representation of the population. Thus the community's concerns for privacy meant we did not have access to identifiable information outside of that provided in anonymous surveys, and instead the building management team was tasked with tracking of participation in the program. Although the same group of participants was invited to respond to the three surveys, a significant portion of respondents chose not to provide their unit numbers. Since this was the only identifying piece of data that we had access to it was 
impossible to reliably match responses to a single apartment unit between the three time points and thus a repeated measures analysis was not feasible. Therefore the central tendency for each question at each time point was treated as an independent sample statistically. It is important to keep in mind, however, that the student housing management team kept track of participation and only those student residents who were participating in the program were sent the surveys. In addition, only the student residents who answered the second survey were sent the third survey. Thus, the responding pool for all three surveys highly overlapped and no residents who were unexposed to the Start Green program responded to the survey.

Since there is debate in the literature on the appropriateness of using parametric tests on ordinal data such as Likert scales, the central tendency of the data was initially analyzed using both parametric and non-parametric tests. Both Kruskal-Wallis and one-way analysis of variance tests were conducted on all the data and the results were compared. There was little difference between the parametric and non-parametric results, thus only the non-parametric data are reported here. Post-hoc tests were performed on significant Kruskal-Wallis results with Dunn's Test using Bonferroni corrections.

\subsection{Methodological Limitations}

As with all Community-based research (CBR) [30,39], the involvement of the participants in the research design introduces possible limitations, such as increased selection and response biases and decreased randomization. However, these limitations are more than balanced by the advantages of this approach, including greater participation rates, decreased drop-out rates over time, increased community trust and engagement, and increased external validity [30,40-43]. Most importantly, CBR is known to foster durable change that is sustainable beyond the application of the intervention [19], which was the main goal of this study. The increased sustainability of CBR based programs has long been observed in public health interventions where it has been argued by many researchers that community involvement in interventions and research not only has the capacity to create durable changes in individual lives but increases the quality of the data overall $[19,30]$. In any case, CBR is an accepted methodology, particularly in applied research where the goal involves understanding how best to advance energy conservation behaviors, for example, rather than solely to test a particular theory where context is less important. In the case of Start Green therefore, part of the program is to intentionally involve the participants in creating their own culture of energy conservation, thus hopefully creating a shift in self and group identity and an increase in the durability of behavioral change.

Another limitation of our approach was the minimal demographic data we were able to collect. It would have been preferable to examine the impacts of many individual characteristics on energy conservation behaviors and attitudes over time, including socio-economic status, religion, ethnicity, gender, age, field of study, employment status of partner, education level of partner, size of family, age of children, first language, country of origin, and length of tenure in Canada. Follow-up studies would benefit from exploring these as covariates to the impact of CBR interventions on longitudinal behavior and attitudes.

\section{Results}

\subsection{Behaviors}

There was a general trend of increasing frequency of reported pro-environmental behaviors (Figure 2), which was shown to be significant in all cases (Table 2). However, this was entirely due to increased self-reported behavior during the intervention (Table 3) and not during the follow-up period (Table 4). Indeed, the trend from closing to follow-up is less clear than between baseline and closing (Figure 2) with some behaviors numerically increasing slightly and others decreasing slightly. Only cold-water laundry changed during the post-intervention period with an additional increase in self-reported frequency. All the other changes during the post-intervention period failed to 
meet statistical significance after pair-wise corrections (Table 3) and thus it is clear that the significant increases in behaviors during the intervention remained elevated during the post-intervention period. The magnitude of the effect depended on the specific behavior. In general all the behaviors increased between 0.5 and 1 point on the Likert scale, generally moving from 'occasionally' to 'often'. The greatest effects were seen in the area of laundry behaviors. For example, participants reported using the cold-water setting on the washing machine less than occasionally (mean rank $=2.67$ ) at baseline whereas at follow-up they reported performing this behavior often (mean rank $=4.03$ ).

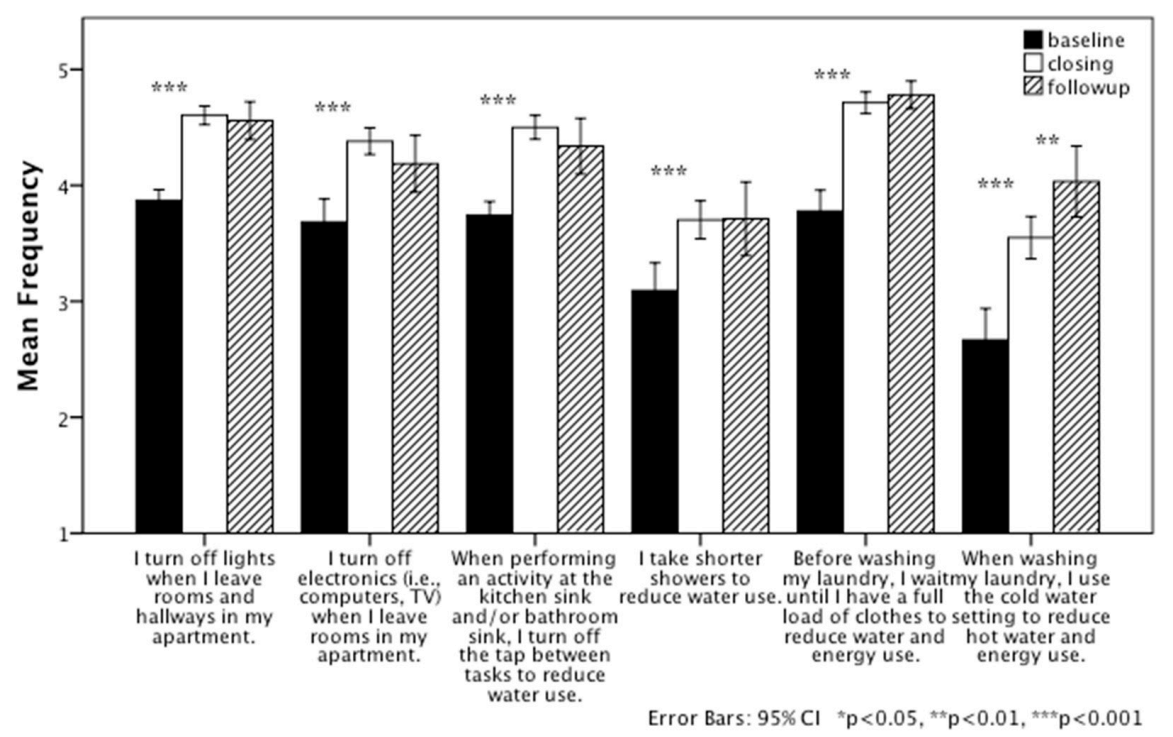

Figure 2. Mean frequency of targeted pro-environmental behaviors during baseline, closing and follow-up time points. Mean frequency is reported on a 5-point Likert scale: never (1); rarely (2); occasionally (3); often (4); and always (5).

Table 2. Kruskal-Wallis analysis of behavioral questions at baseline, closing and follow-up. Degrees of freedom (df), sample size $(\mathrm{N})$, chi square $\left(\chi^{2}\right)$, and $p$ value are reported.

\begin{tabular}{lcccc}
\hline $\begin{array}{l}\text { Questions } \\
\text { Please Indicate How Often You do the Following: }\end{array}$ & df & $\mathbf{N}$ & $\chi^{2}$ & $p$ \\
\hline I turn off lights when I leave rooms ... & 2 & 319 & 71.027 & $<0.01$ \\
I turn off electronics when I leave rooms ... & 2 & 316 & 39.505 & $<0.01$ \\
I turn off the tap between tasks ... & 2 & 317 & 57.227 & $<0.01$ \\
I take shorter showers ... & 2 & 317 & 18.734 & $<0.01$ \\
I wait for a full load of laundry ... & 2 & 316 & 117.503 & $<0.01$ \\
When doing laundry I use the cold water setting... & 2 & 317 & 40.221 & $<0.01$ \\
\hline
\end{tabular}

Table 3. Baseline to closing pairwise comparison of self-reported behavior.

\begin{tabular}{lccccc}
\hline $\begin{array}{l}\text { Questions } \\
\text { Please Indicate How Often You do the Following: }\end{array}$ & df & $\mathbf{N}$ & $\chi^{2}$ & $p$ \\
\hline I turn off lights when I leave rooms ... & 1 & 260 & 69.170 & $<0.01$ \\
I turn off electronics when I leave rooms ... & 1 & 257 & 40.527 & $<0.01$ \\
I turn off the tap between tasks ... & 1 & 258 & 56.049 & $<0.01$ \\
I take shorter showers ... & 1 & 258 & 16.747 & $<0.01$ \\
I wait for a full load of laundry ... & 1 & 257 & 104.023 & $<0.01$ \\
When doing laundry I use the cold water setting... & 1 & 258 & 25.393 & $<0.01$ \\
\hline
\end{tabular}


Table 4. Closing to Follow-up pairwise comparison of self-reported behaviors.

\begin{tabular}{lccccc}
\hline $\begin{array}{l}\text { Questions } \\
\text { Please Indicate How Often You do the Following: }\end{array}$ & df & $\mathbf{N}$ & $\chi^{2}$ & $p$ \\
\hline I turn off lights when I leave rooms ... & 1 & 265 & 0.149 & $>0.05$ \\
I turn off electronics when I leave rooms ... & 1 & 262 & 2.145 & $>0.05$ \\
I turn off the tap between tasks ... & 1 & 263 & 1.264 & $>0.05$ \\
I take shorter showers ... & 1 & 263 & 0.072 & $>0.05$ \\
I wait for a full load of laundry ... & 1 & 262 & 0.015 & $>0.05$ \\
When doing laundry I use the cold water setting... & 1 & 263 & 7.674 & $<0.05$ \\
\hline
\end{tabular}

\subsection{Perceived Behavioral Importance}

There were no clear numerical trends over the three time periods. In some cases it even appears that the intervention period led to a decrease in perceived importance of target behaviors (Figure 3). The only significant change was seen in the cold-water washes behavioral domain (Table 5). However, pairwise comparisons and corrections revealed that this increase in perceived importance occurred during the post-intervention period, $\chi^{2}(1, \mathrm{~N}=263)=2.67, p=0.022$, not during the intervention, $\chi^{2}(1, \mathrm{~N}=256)=0.26, p=0.78$. This is in agreement with the behavioral data, which also suggested that the largest effects were on the cold-water washes target.

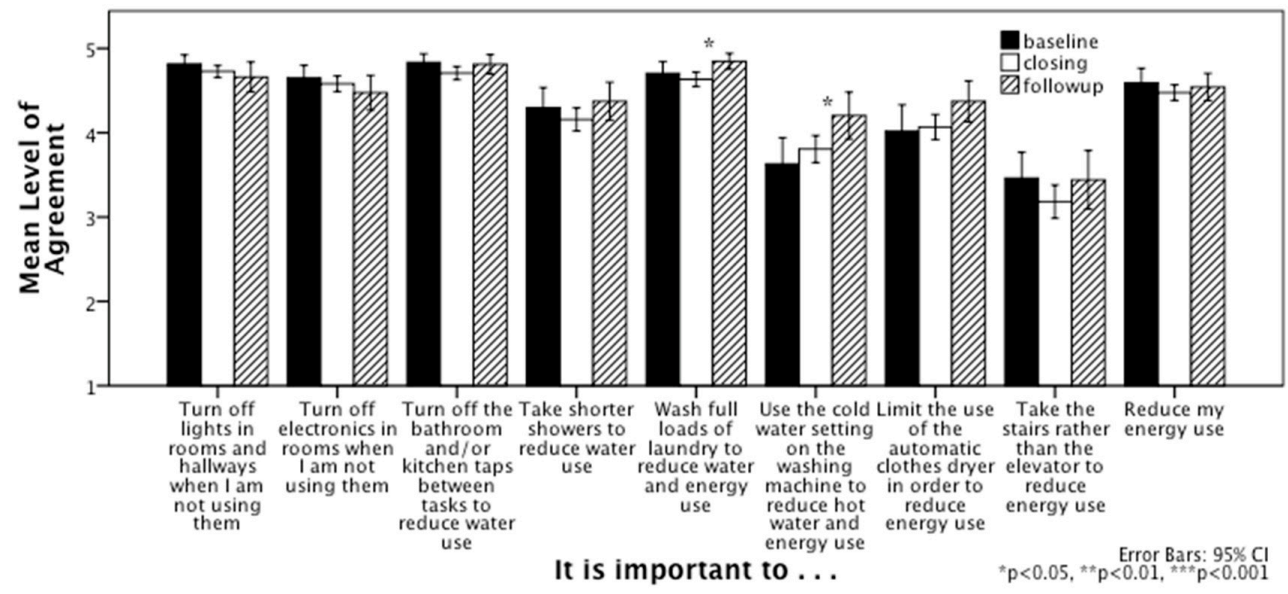

Figure 3. Mean level of agreement to statements of behavioral importance at baseline, closing and follow-up time points. The perceived importance of target behaviors was self-reported using a 5 point Likert scale: strongly disagree (1); disagree (2); neither agree or disagree (3); agree (4); and strongly agree (5).

Table 5. Kruskal-Wallis analysis of statements on perceived importance of target behaviors at baseline, closing and follow-up. Degrees of freedom (df), sample size (N), chi-square $\left(\chi^{2}\right)$, and $p$ values are reported for all questions.

\begin{tabular}{lcccc}
\hline $\begin{array}{l}\text { Questions } \\
\text { Please Indicate Your Level of Agreement with the Following: It is Important to }\end{array}$ & df & $\mathbf{N}$ & $\chi^{\mathbf{2}}$ & $\boldsymbol{p}$ \\
\hline Turn off lights when I leave rooms . . . & 2 & 316 & 1.420 & $>0.05$ \\
Turn off electronics when I leave rooms . . & 2 & 314 & 1.015 & $>0.05$ \\
Turn off the tap between tasks ... & 2 & 313 & 3.665 & $>0.05$ \\
Take shorter showers ... & 2 & 314 & 2.735 & $>0.05$ \\
Wash full load of laundry ... & 2 & 311 & 5.375 & $>0.05$ \\
Use the cold water setting on the laundry machine... & 2 & 315 & 10.221 & $<0.01$ \\
Limit the use of the dryer ... & 2 & 315 & 4.781 & $>0.05$ \\
Take the stairs rather than the elevator .... & 2 & 314 & 2.757 & $>0.05$ \\
Reduce my energy use & 2 & 314 & 1.987 & $>0.05$ \\
\hline
\end{tabular}




\subsection{Perceived Family Norms}

There were no consistent numerical trends of reported family norms across the three time points (Figure 4). The perceived family expectation to use cold-water washes was the only norm to increase during the study (Table 6), and as seen above, this only increased significantly during the post-intervention period, $\chi^{2}(1, \mathrm{~N}=258)=2.43, p=0.04$, not during the intervention, $\chi^{2}(1, N=251)=0.31, p=0.95$.

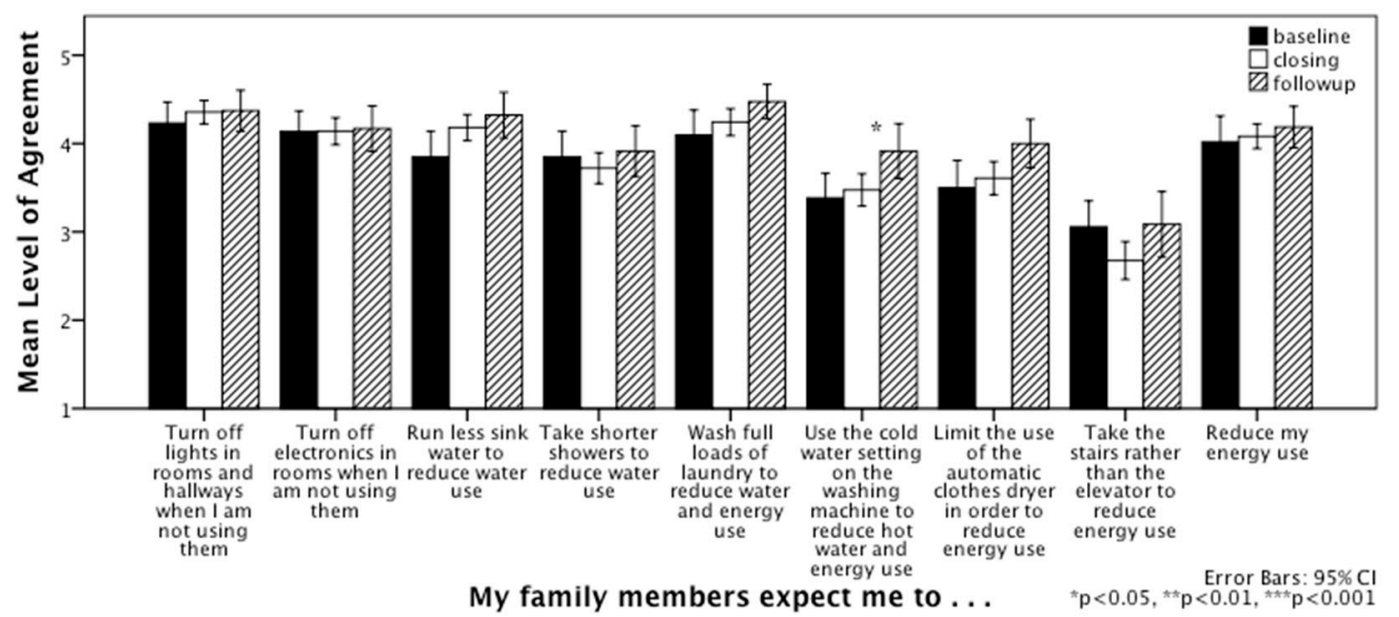

Figure 4. Mean level of agreement to statements of family expectations of target behaviors at baseline, closing and follow-up time points. Perceived family expectations were self-reported using a 5 point Likert scale ranging from strongly disagree (1) to strongly agree (5).

Table 6. Kruskal-Wallis analysis of agreement with statements on the perceived family expectations surrounding target behaviors at baseline, closing and follow-up. Degrees of freedom (df), sample size (N), chi-square $\left(\chi^{2}\right)$, and $p$ values are reported.

\begin{tabular}{|c|c|c|c|c|}
\hline $\begin{array}{l}\text { Questions } \\
\text { Please Indicate Your Level of Agreement with the Following: } \\
\text { My Family Members Expect Me to }\end{array}$ & df & $\mathbf{N}$ & $x^{2}$ & $p$ \\
\hline Turn off lights when I leave rooms .. & 2 & 312 & 2.268 & $>0.05$ \\
\hline Turn off electronics when I leave rooms ... & 2 & 309 & 0.587 & $>0.05$ \\
\hline Turn off the tap between tasks ... & 2 & 310 & 9.223 & $>0.05$ \\
\hline Take shorter showers ... & 2 & 309 & 0.863 & $>0.05$ \\
\hline Wash full load of laundry ... & 2 & 309 & 4.598 & $>0.05$ \\
\hline Use the cold water setting on the laundry machine... & 2 & 310 & 8.383 & $<0.01$ \\
\hline Limit the use of the dryer... & 2 & 309 & 5.794 & $>0.05$ \\
\hline Take the stairs rather than the elevator. & 2 & 310 & 5.275 & $>0.05$ \\
\hline Reduce my energy use & 2 & 309 & 0.624 & $>0.05$ \\
\hline
\end{tabular}

\subsection{Perceived Behavioral Convenience}

Although the perceived convenience of most of the target behaviors was not altered during the study, (Figure 5), the perceived convenience of using cold-water washes significantly increased (see Table 7). However, pairwise corrections showed that the perceived ease of using cold water washes was only significant during the post-intervention period, between baseline and follow-up $\chi^{2}(1, N=257)=2.83, p=0.013$. There were no significant changes in perceived convenience during the intervention (cold-water washes: $\left.\chi^{2}(1, \mathrm{~N}=250)=1.20, p=0.69\right)$. 
Table 7. Kruskal-Wallis analysis of perceived convenience of target behaviors at baseline, closing and follow-up. Degrees of freedom (df), sample size $(\mathrm{N})$, chi-square $\left(\chi^{2}\right)$, and $p$ values are reported.

\begin{tabular}{lrrrr}
\hline $\begin{array}{l}\text { Questions } \\
\text { Please Indicate Your Level of Agreement with the Following: I Find it Easy to }\end{array}$ & $d f$ & $\mathbf{N}$ & $\chi^{2}$ & $p$ \\
\hline Turn off lights when I leave rooms . . . & 2 & 309 & 0.768 & $>0.05$ \\
Turn off electronics when I leave rooms ... & 2 & 310 & 0.058 & $>0.05$ \\
Turn off the tap between tasks ... & 2 & 308 & 1.107 & $>0.05$ \\
Take shorter showers ... & 2 & 308 & 1.097 & $>0.05$ \\
Wash full loads of laundry ... & 2 & 309 & 0.321 & $>0.05$ \\
Use the cold water setting on the laundry machine... & 2 & 309 & 8.735 & $<0.05$ \\
Limit the use of the dryer ... & 2 & 310 & 3.887 & $>0.05$ \\
Take the stairs rather than the elevator .... & 2 & 309 & 4.509 & $>0.05$ \\
Reduce my energy use & 2 & 310 & 6.829 & $<0.05$ \\
\hline
\end{tabular}

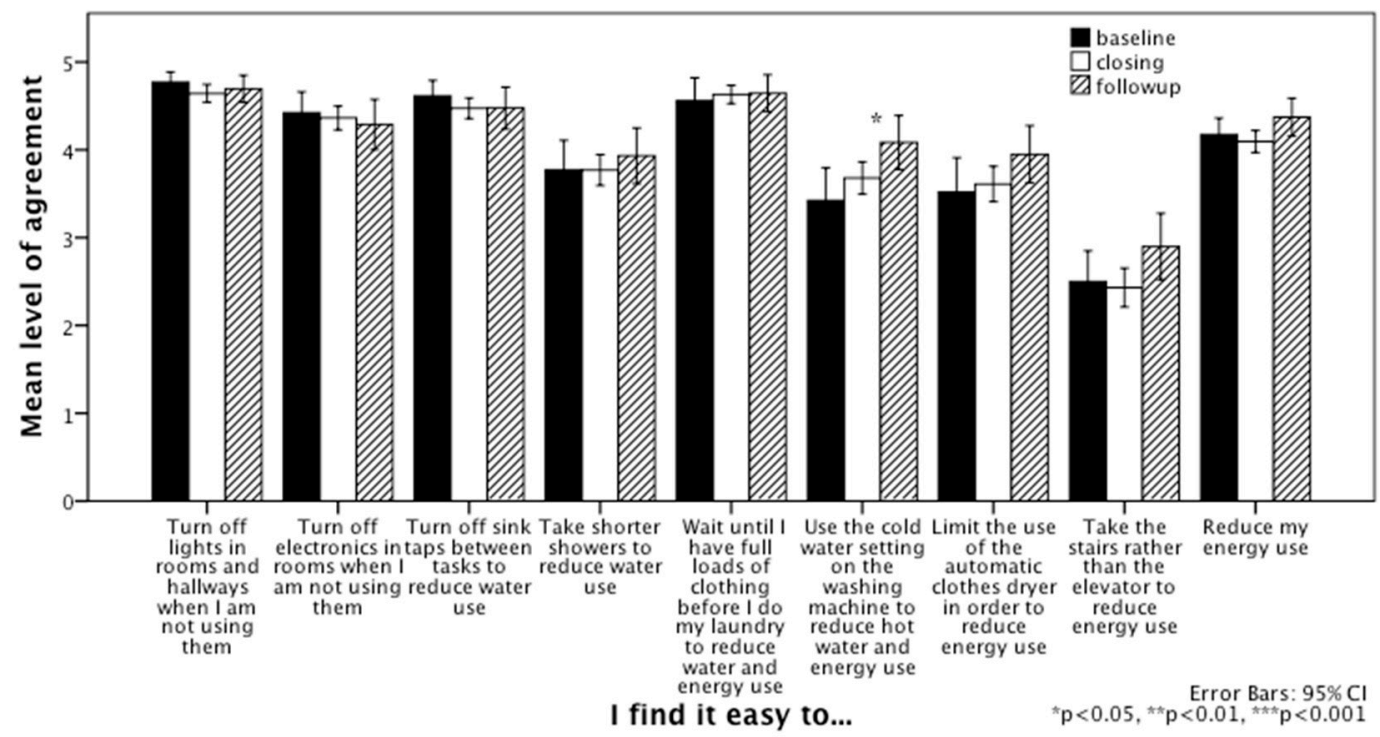

Figure 5. Mean level of agreement to statements of behavioral convenience at baseline, closing and follow-up time points. Perceived convenience was self-reported using a 5 point Likert scale ranging from strongly disagree (1) to strongly agree (5).

\subsection{Generalized Pro-Conservation Decision Making}

There is a significant positive trend of increasing consideration of environmental concerns during general decision making in the studied population $\chi^{2}(2, \mathrm{~N}=327)=22.699, p<0.001$ (Figure 6). Reported conversational decision making significantly increased during the intervention, $\chi^{2}(1, N=268)=3.27, p<0.001$, and significantly increased again during the post-intervention period, $\chi^{2}(1, N=271)=2.69, p=0.02$. This continuous increase during both phases of the study is in agreement with the theory that the practice of external behaviors can change internalized attitudes and values, and a sense of identity [3]. 


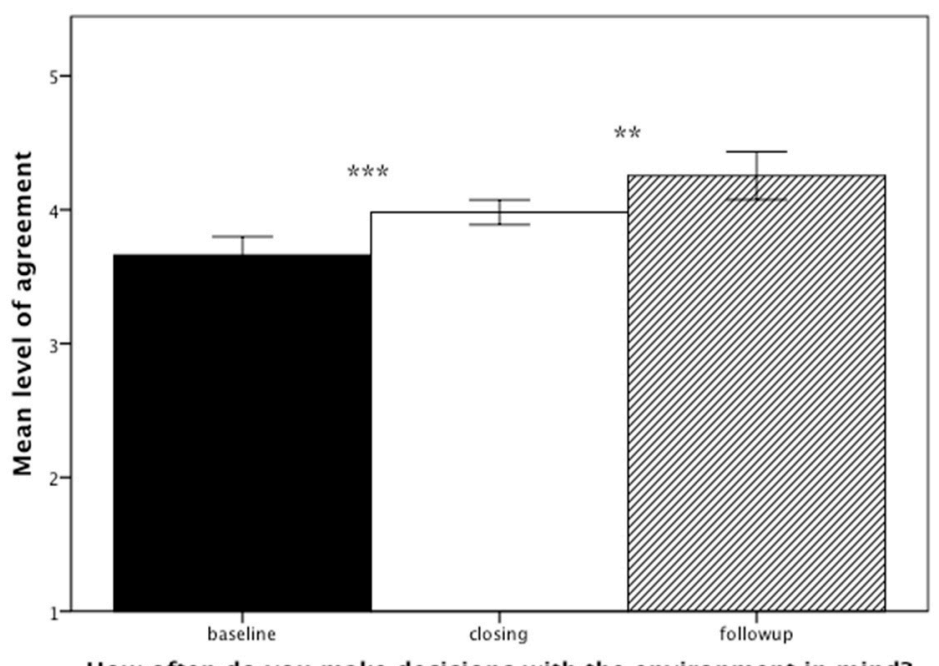

How often do you make decisions with the environment in mind? $" p<0.05, * p<0.01, * " p<0.001$

Figure 6. Mean level of agreement to a statement indicating generalized conservation decision making at baseline, closing and follow-up time points. This generalized value was self-reported using a 5 point Likert scale ranging from strongly disagree (1) to strongly agree (5).

\section{Discussion}

\subsection{Behavioral Persistence}

By utilizing workshops, peer mentoring, group action, public commitment, and prompts the Start Green program successfully increased self-reported pro-environmental behaviors across a wide range of household energy conservation activities. At program closing all the measured behaviors had significantly increased as compared to baseline. The largest increases were in the areas of cold-water laundry washes, water conservation, and electricity use. These short-term results are in agreement with the majority of the literature, which has documented that public commitment and social norms are often quite effective in creating significant short-term improvements in pro-environmental behaviors [1]. During the 7-month follow-up phase there were no significant changes as compared to program closing, suggesting that the individuals had made durable pro-environmental behavioral changes that had persisted long-term without further intervention. These results are in agreement with the theoretical literature, which postulates that techniques that create an internal change, or a personal norm, such as a commitment, or perceived social norms should be better at creating persistence than more external techniques such as incentives [3]. This is an encouraging result and suggests that the post-intervention reversion to baseline trend observed in multiple long-term studies may be due to intervention design rather than an inability of participants to maintain long-term pro-environmental behaviors. This contributes to the growing literature suggesting that public commitments, social norms, and peer mentoring, along with tailored information and prompts can be effective in creating robust pro-environmental behaviors that persist beyond the termination of the intervention.

Because this study was limited to self-reported behaviors due to the practical complexities of gathering energy usage data (see methods section), it is possible that the results do not reflect actual energy conservation [44]. It would have been ideal to collect direct data on energy usage and compare that to self-reports. However, even if the self-reported behavioral improvement we see here is more a reflection of behavioral intention, rather than actual increased conservation and energy savings, it is still clear that there has been a significant long-term change in this population. Self-reported pro-environmental behaviors are often used in field evaluations and it is rare to observe self-reported long-term post-intervention persistence [1]. Thus, there doesn't seem to be a strong trend of socially acceptable self-reporting in the literature, at least not beyond the termination of 
the intervention program. These results are therefore a rare example of self-reported persistence of increased pro-environmental behaviors.

In addition, it may be tempting to attribute the results to response, or experimenters' bias given the highly social and participatory nature of the program. As mentioned in the methods section, although there are many benefits of the participation aspect of CBR, this can also introduce possible confounds. In the case of Start Green, the participation of a small number of student residents in planning the intervention (via focus groups) and implementing the intervention (via organizing events and acting as floor mentors) might have not only increased motivation to change behaviors, but may also have increased response bias on the surveys. Because Start Green targeted social norms directly, in an attempt to foster a strong sense of community responsibility, it is reasonable to question that this sense of responsibility might confound self-reports of behaviors, values, attitudes and especially social norms. However, this is not what was observed, as only self-reported behaviors increased. Although the program directly promoted the importance of the target energy conservation behaviors, created social norm pressures, and provided tools to overcome behavioral obstacles, the self-reported perceived importance, family norms, and behavioral convenience did not increase during the intervention. If the results were due to response or experimenters' bias, even unconsciously, we would expect to see significant increases across the board, or at least in most categories. Since the behavior questions were not qualitatively different from the other questions in terms of transparency, it should have been equally clear to the respondents what the 'right' answer was in each section. However, many of the perceived behavioral importance questions for example numerically decreased during the intervention period (see Figure 3) in direct opposition to the expected response/experimenter bias trend. It is thus unlikely that the reported increases in behaviors are due to biases created by the participation in the design of the intervention.

As mentioned in the methods section the lack of depth of individual demographic data introduced some limitations. For example it would be reasonable to question if cost and/or changes in socio-economic status has an impact on energy conservation. In this particular population, though, as the majority of the population were graduate students, it is unlikely that their socio-economic status changed significantly during the course of the study. In addition, because increased conservation behaviors did not have an impact on the residents' monthly costs the results we see here can not be attributed to cost motivations. Other demographics might have had an impact. For example, in this population, it would have been especially interesting to explore the impact of country of origin, and length of tenure in Canada on energy conservation behaviors. There is evidence that a change in residence or being exposed to a new culture can increase willingness to try new behaviors or life-styles. As a large proportion of the participants in Start Green were international students this may have been a significant contributing factor to our results. As CBR intervention design is tailored to a specific population, understanding the characteristics of each community is more important than if applying a more formulaic approach. Thus, future studies would benefit from tracking detailed individual demographics in order to tease apart the aspects of the program that are effective when combined with specific population characteristics and thus make it possible for this approach to be applied to different populations successfully.

It also might be tempting to attribute the reported behavior changes to the seasonal changes between baseline and closing (September to February), as seasonal changes are often important factors in energy conservation behaviors. However, since February is often colder than September in Toronto, Canada, and since the follow-up survey took place in September one year after the September baseline measurements, it is reasonable to suggest that differences between baseline, closing and follow-up conservation behaviors are not due to temperature or seasonal differences and thus can be attributed, at least in part, to the interventions. Taken together with the large sample sizes and the strength of the effects, this study strongly suggests that the Start Green interventions not only increased pro-environmental behaviors, but also led to the long-term persistence of those behaviors. These results contribute to the growing evidence [20] that behavioral interventions that 
utilize multiple CBR techniques such as commitments and mentorship, may be effective at creating persistent behavior change.

Future research on persistence of behavior change would benefit from increased exploration of demographics to identify any covariates and gain more clarity on the drivers of behavior change. In addition, the matching of pre and post surveys alongside direct energy consumption measures will be necessary to conclude that these self-reports translate into impactful energy conservation.

\subsection{Behavior vs. Perceived Importance, Convenience and Family Norms}

We were also interested in how perceived importance, convenience, and family expectations of the target behaviors varied with behavioral practice. During the intervention there were no significant changes in any of these measures. These data suggest that despite the program reinforcing the importance of these behaviors, and despite providing tools to reduce the behavioral obstacles of performing the behaviors, this did not have an immediate effect on the attitudes of the population. In addition, family expectations did not change as a result of the social norm techniques. One of the limitations of the study was that participants were somewhat self-selecting as previously discussed in the methods section. Thus, it is possible that the intervention did not increase perceived importance, convenience and family expectations because the participants had already reached a pro-environmental maximum. However, the significant post-intervention increases in these categories on the importance, convenience and family expectations of using cold water washes suggest that this is not the case. Interestingly, the fact that these attitudes did change during the post-intervention period suggests that the practice of behaviors in the absence of intervention may contribute to increasing the perceived importance, convenience and family expectations to perform the target behaviors.

These results also provide some insight to the complex relationship between attitudes and behavior. It is often assumed that attitude change is a necessary prerequisite to durable behavioral change, however there has been increasing evidence that it is possible to directly impact reported behaviors without changing attitudes first, and that this may be a more effective route to persistent behavioral change [12]. Indeed, these results suggest that significant changes in perceived importance, convenience or family expectations are not necessary pre, or even co-requisites to pro-environmental behavioral changes, at least in the short-term. It is possible that changes in these perceptions or attitudes contribute to the persistence of related behaviors over a longer time frame, however. In addition these results suggest the possibility that changing behaviors first can actually, over time, lead to changes in perceptions of importance, convenience and family expectations in specific areas. This interesting result also suggests that it may be possible to further increase pro-environmental attitudes even in already highly pro-environmental populations, but that it may occur over a longer timeline.

This ability of a behavioral practice to change attitudes is in agreement with the concept of cognitive dissonance. When a mismatch between behaviors and attitudes becomes salient to an individual he or she experiences a feeling of discomfort, or cognitive dissonance, and is thus motivated to either change his or her behavior, or change his or her attitudes in order to regain some consistency [45]. Often, research has focused on this phenomenon from the attitude change side of the equation. However, these results show that it is also possible for people to do the latter and change their attitudes to be more consonant with their behaviors. It is tempting again to conclude that this population already had, on average, positive attitudes towards pro-environmental behavior, and that the intervention simply acted to remind people, and thus create salient dissonance, that their behaviors were not in line with their pre-existing attitudes. This would explain the significant increase in behaviors and the lack of significant attitude change during the intervention period. However, this doesn't explain the increase in attitudes about cold-water use and full loads of laundry observed during the follow-up period. Thus, these results suggest that the relationship between target behaviors and perceived importance, convenience and family expectations of those behaviors is more complex than often reported, and that targeting behaviors directly without changing these attitudes can result in durable behavior change that persists post-intervention. 
In addition, these results also align with self-perception theory, which suggests that individuals infer their internal states, such as attitudes and emotions, from observing their behavior over time [46]. This would suggest that the more people practiced the target energy conservation behaviors, the more likely they were to view themselves as individuals who conserve energy, thus changing some of their attitudes and sense of group membership over time and practice. This has been observed in other pro-environmental studies where past pro-environmental behaviors influence present pro-environmental social identity [47].

It may be tempting, based on our results and these related theories to encourage future interventions to avoid putting resources into changing attitudes, family norms, or conveniences. However, because findings from CBR are irrevocably linked to the culture and social norms of the specific population in question, it would be necessary for future studies to test if our results can be replicated in other populations with different characteristics. In addition, performing preliminary investigations on the specific barriers to target behaviors, and inviting the community to participate in the intervention design is an important part of CBR approaches [20]. The trust and commitment that is fostered by inviting the community to self-determine the program is possibly the key element in the increased willingness to try new behaviors despite a lack of increase in perceived importance, convenience or family pressure. Thus future studies might benefit from testing how differing levels of community participation interact with community demographics and attitudes in fostering durable behavior change.

\subsection{Generalized Conservation Behavior}

In the interest of keeping the surveys as short as possible and thus the participation rate high, it was necessary to use a single general question to explore this area, although stronger results would have emerged from multiple specific questions about behaviors in non-target behavioral domains such as recycling or buying green products. The frequency of general pro-environmental decision making significantly increased at closing and then again at follow-up suggesting that both the intervention and the post-intervention practice had an impact. This result suggests that environmental decision-making became more generalized over the course of the study and thus is likely to be applied outside of the target domains and to a wide range of decisions. This finding indicates that the ongoing pro-environment behaviors following the end of the intervention likely contributed not only to the change in attitudes described above, but also, potentially, to the proliferation of pro-environmental behaviors to others beyond those targeted in this intervention. It would be fascinating to track other pro environmental behaviors not targeted by the intervention, to identify whether persistence of the targeted behavior can influence proliferation to related behaviors.

\section{Conclusions}

The long-term evaluation of the Start Green program revealed both short and long-term increases in pro-environmental behaviors, which persisted seven months beyond the end of the intervention. In addition, generalized pro-environmental decision making increased continuously alongside the specific target behaviors over the long-term suggesting a generalized conservation approach, which could contribute to the proliferation of conservation behaviors to non-target domains. The Start Green techniques appear to have directly altered self-reported behaviors during the intervention and indirectly impacted the perceived importance, convenience and family expectations around some of the target behaviors after a period of behavioral practice. This practice may eventually create an internal, as well as an external, norm thus only impacting these attitudes after a period of time.

Further research that tracks more detailed demographics including geographical, social, political and historical data, a more robust set of pro-environmental values and decisions, as well as behaviors not targeted by the initial intervention would contribute to the understanding of these trends towards persistence as well as possible proliferation of pro-environmental behaviors. Studies that use a more qualitative approach would also be useful in order to provide more insight into any possible biases 
created by CRB participation and/or how the sense of self evolves during the interventions and impacts the outcomes. These hopeful results emphasize the combination of social and individual motivators of changed behavior, and further research could explore this combination of factors, through interdisciplinary approaches drawing on expertise in both psychology and sociology. For example, research approaches stressing participant narratives and focus groups to explore personal experiences of behavior change and the meanings and motivations that underlie them could provide a fresh perspective on the drivers of behavior change seen here. It would be useful to track behaviors and attitudes at an individual level within families to explore the impact children's habits and attitudes have on their parents during CRB and visa versa. It would also be fascinating to explore the extent of potential proliferation to other energy conserving actions. For example, could changes in household energy use ultimately influence transportation behaviors, or diet? In addition, a more structured approach to the coincident monitoring of household energy use through individualized billing, combined with matched pre, post and follow up surveys could confirm the actual impact of reported energy conservation behaviors and quantify the effect size.

These are encouraging preliminary results that suggest that well designed CRB interventions, which utilize social norms and public commitments, can produce durable pro-environmental behaviors that extend beyond the targeted activities and persist after the removal of the intervention.

Acknowledgments: This research was supported in part by a University of Toronto Excellence Award and The Social Sciences and Humanities Research Council of Canada. Start Green was funded by Ontario Ministry of the Environment. Many University of Toronto Sustainability Office staff and volunteers supported the preparation, delivery and evaluation of Start Green. Stuart Chan, Elah Feder, Tyler Hunt and Leah Sumnauth MacIntosh all were involved in the development and supervision of the interventions. Gloria Cuneo and Shawn Mitchell at Student Family Housing contributed to their delivery. Zannah Matson made a significant contribution to the development of the ideas presented in this article. Birsen Donmez, Tyler Hunt, Stuart Chan, and Ray Tomalty generously edited and reviewed this manuscript prior to submission. We are grateful to anonymous reviewers for their constructive comments and suggestions.

Author Contributions: S. Barnett Burns collected, analyzed and presented the data reported above and wrote the bulk of this article. Beth Savan originated and supervised the research project, including the design and data collection, wrote some of the manuscript, edited the parts she did not write and contributed to the revisions.

Conflicts of Interest: The authors declare no conflict of interest.

\section{References}

1. Abrahamse, W.; Steg, L.; Vlek, C.; Rothengatter, T. A review of intervention studies aimed at household energy conservation. J. Environ. Psychol. 2005, 25, 273-291. [CrossRef]

2. Delmas, M.; Fischlein, M.; Asensio, O. Information Strategies and Energy a Meta-Analysis of Experimental Studies. Energy Policy 2013, 61, 729-739. [CrossRef]

3. De Young, R. Changing Behavior and Making it Stick: The Conceptualization and Management of Conservation Behavior. Environ. Behav. 1993, 25, 485-505. [CrossRef]

4. Dietz, T.; Gardner, G.T.; Gilligan, J.; Stern, P.C.; Vandenbergh, M.P. Household actions can provide a behavioral wedge to rapidly reduce US carbon emissions. Proc. Natl. Acad. Sci. USA 2009, 106, 18452-18456. [CrossRef] [PubMed]

5. Dwyer, W.O.; Leeming, F.C.; Cobern, M.K.; Porter, B.E.; Jackson, J.M. Critical Review of Behavioral Interventions to Preserve the Environment: Research Since 1980. Environ. Behav. 1993, 25, 275-321. [CrossRef]

6. Geller, E.S.; Geller, E.S. Applied behavior analysis and social marketing: An integration for environmental preservation. J. Soc. Issues 1989, 45, 17-36. [CrossRef]

7. Hayes, S.C.; Cone, J.D. Reduction of residential consumption of electricity through simple monthly feedback. J. Appl. Behav. Anal. 1981, 14, 81-88. [CrossRef] [PubMed]

8. Massung, E.; Coyle, D.; Cater, K.F.; Jay, M.; Preist, C. Using crowdsourcing to support pro-environmental community activism. In Proceedings of the SIGCHI Conference on Human Factors in Computing Systems-CHI '13, Paris, France, 27 April-2 May 2013; p. 371.

9. Chawla, L. Significant life experiences revisited: A review of research on sources of environmental sensitivity. J. Environ. Educ. 1998, 29, 11-21. [CrossRef] 
10. Lo, S.H.; van Breukelen, G.J.P.; Peters, G.J.Y.; Kok, G. Proenvironmental travel behavior among office workers: A qualitative study of individual and organizational determinants. Transp. Res. Part A Policy Pract. 2013, 56, 11-22. [CrossRef]

11. Katzev, R.; Johnson, T.R. Comparing the Effects of Monetary Incentives and Foot-in-the-Door Strategies in Promoting Residential Electricity Conservation. J. Appl. Soc. Psychol. 1984, 14, 12-27. [CrossRef]

12. Mckenzie-Mohr, D. Promoting Sustainable Behavior: An Introduction to Community-Based Social Marketing. J. Soc. Issues 2000, 56, 543-554. [CrossRef]

13. Pichert, D.; Katsikopoulos, K.V. Green defaults: Information presentation and pro-environmental behaviour. J. Environ. Psychol. 2008, 28, 63-73. [CrossRef]

14. Wallenborn, G.; Orsini, M.; Vanhaverbeke, J. Household appropriation of electricity monitors. Int. J. Consum. Stud. 2011, 35, 146-152. [CrossRef]

15. Kappel, K.; Grechenig, T. "Show-Me": Water Consumption At a Glance To Promote Water Conservation in the Shower. In Proceedings of the 4th International Conference on Persuasive Technology, Claremont, CA, USA, 26-29 April 2009. [CrossRef]

16. Gronhoj, A.; Thogersen, J. Feedback on household electricity consumption: Learning and social influence processes. Int. J. Consum. Stud. 2011, 35, 138-145. [CrossRef]

17. Katzev, R.; Bachman, W. Effects of deferred payment and fare manipulations on urban bus ridership. J. Appl. Psychol. 1982, 67, 83-88. [CrossRef]

18. Wang, T.; Katzev, R. Group Commitment and Resource Conservation: Two Field Experiments on Promoting Recycling. J. Appl. Soc. Psychol. 1990, 265-275. [CrossRef]

19. Altman, D.G. Sustaining interventions in community systems: On the relationship between researchers and communities. Health Psychol. 1995, 14, 526-536. [CrossRef] [PubMed]

20. McKenzie-Mohr, D. Fostering sustainable behavior through community-based social marketing. Am. Psychol. 2000, 55, 531-537. [CrossRef] [PubMed]

21. Burn, S.M.; Oskamp, S. Increasing Community Recycling with Persuasive Communication and Public Commitment. J. Appl. Soc. Psychol. 1986, 16, 29-41. [CrossRef]

22. Lokhorst, A.M.; van Dijk, J.; Staats, H.; van Dijk, E.; de Snoo, G. Using tailored information and public commitment to improve the environmental quality of farm lands: An example from the Netherlands. Hum. Ecol. 2010, 38, 113-122. [CrossRef] [PubMed]

23. Osbaldiston, R.; Sheldon, K.M. Promoting internalized motivation for environmentally responsible behavior: A prospective study of environmental goals. J. Environ. Psychol. 2003, 23, 349-357. [CrossRef]

24. Staats, H.; Harland, P.; Wilke, H.A.M. Effecting Durable Change: A Team Approach to Improve Environmental Behavior in the Household. Environ. Behav. 2004, 36, 341-367. [CrossRef]

25. Werner, C.M.; Makela, E. Motivations and Behaviors That Support Recycling. J. Environ. Psychol. 1998, 18, 373-386. [CrossRef]

26. Allcott, H. Social norms and energy conservation. J. Public Econ. 2011, 95, 1082-1095. [CrossRef]

27. Brandon, G.; Lewis, A. Reducing Household Energy Consumption: A Qualitative and Quantitative Field Study. J. Environ. Psychol. 1999, 19, 75-85. [CrossRef]

28. Carrico, A.R.; Riemer, M. Motivating energy conservation in the workplace: An evaluation of the use of group-level feedback and peer education. J. Environ. Psychol. 2011, 31, 1-13. [CrossRef]

29. Schelly, C.; Cross, J.; Franzen, W.; Hall, P.; Reeve, S. Creating a conservation culture: Reducing energy consumption in a public high school. Environ. Behav. 2011, 43, 316-343. [CrossRef]

30. Goodman, M.S.; Thompson, V.L.S.; Arroyo-Johnson, C.; Gennarelli, R.; Drake, B.F.; Bajwa, P.; Witherspoon, M.; Bowen, D. Evaluating community engagement in research: Quantitative measure development. J. Commun. Psychol. 2017, 45, 17-32. [CrossRef] [PubMed]

31. Tajfel, H.; Turner, J.C. The social identity theory of intergroup behavior. In Key Readings in Social Psychology. Political Psychology: Key Readings; Jost, J.T., Sidanius, J., Eds.; Psychology Press: New York, NY, USA, 2004; pp. 276-293.

32. Bamberg, S.; Rees, J.; Seebauer, S. Collective climate action: Determinants of participation intention in community-based pro-environmental initiatives. J. Environ. Psychol. 2015, 43, 155-165. [CrossRef]

33. Janis, I.L.; Rodin, J. Attribution, control, and decision-making: Social psychology and health care. In Health Psychology; Stone, G., Cohen, F., Adler, N., Eds.; Jossey-Bass: San Francisco, CA, USA, 1979; pp. 487-521. 
34. Bracht, N.; Tsouros, A. Principles and strategies of effective community participation. Health Promot. Int. 1990, 5, 199-208. [CrossRef]

35. Bandura, A. Social Foundations of Thought and Action: A Social Cognitive Theory; Prentice-Hall: Bergen County, NJ, USA, 1986.

36. Rappaport, J. Terms of Empowerment/Exemplars of Prevention: Toward a Theory for Community Psychology 1. Am. J. Community Psychol. 1987, 15, 121-148. [CrossRef] [PubMed]

37. Stokes, L.C.; Mildenberger, M.; Savan, B.; Kolenda, B. Analyzing Barriers to Energy Conservation in Residences and Offices: The Rewire Program at the University of Toronto. Appl. Environ. Educ. Commun. 2012, 11, 88-98. [CrossRef]

38. Mildenberger, M.; Stokes, L.; Savan, B.; Kolenda, B.; Dolderman, D. Environmental Reviews and Case Studies: Beyond the Information Campaign: Community-Based Energy Behavioral Change at the University of Toronto. Environ. Pract. 2013, 15, 147-155. [CrossRef]

39. Savan, B.; Sider, D. Contrasting Approaches to Community-based Research and a Case Study of Community Sustainability in Toronto, Canada. Local Environ. 2003, 8, 303-316. [CrossRef]

40. Estreet, A.; Apata, J.; Kamangar, F.; Schutzman, C.; Buccheri, J.; O'Keefe, A.-M.; Wagner, F.; Sheikhattari, P. Improving Participants' Retention in a Smoking Cessation Intervention Using a Community-based Participatory Research Approach. Int. J. Prev. Med. 2017, 8, 106. [CrossRef] [PubMed]

41. Jagosh, J.; Macaulay, A.C.; Pluye, P.; Salsberg, J.; Bush, P.L.; Henderson, J.; Sirett, E.; Wong, G.; Cargo, M.; Herbert, C.P.; et al. Uncovering the benefits of participatory research: Implications of a realist review for health research and practice. Milbank Q. 2012, 90, 311-346. [CrossRef] [PubMed]

42. Jagosh, J.; Pluye, P.; Macaulay, A.C.; Salsberg, J.; Henderson, J.; Sirett, E.; Bush, P.L.; Seller, R.; Wong, G.; Greenhalgh, T.; et al. Assessing the outcomes of participatory research: Protocol for identifying, selecting, appraising and synthesizing the literature for realist review. Implement. Sci. 2011, 6, 24. [CrossRef] [PubMed]

43. De las Nueces, D.; Hacker, K.; DiGirolamo, A.; Hicks, L.S. A systematic review of community-based participatory research to enhance clinical trials in racial and ethnic minority groups. Health Serv. Res. 2012, 47, 1363-1386. [CrossRef] [PubMed]

44. Hiramatsu, A.; Kurisu, K.; Hanaki, K. Environmental Consciousness in Daily Activities Measured by Negative Prompts. Sustainability 2016, 8, 24. [CrossRef]

45. Festinger, L. Cognitive dissonance. Sci. Am. 1962, 207, 93-102. [CrossRef] [PubMed]

46. Bem, D.J. Self-Perception Theory. Adv. Exp. Soc. Psychol. 1972, 6, 1-62.

47. Van der Werff, E.; Steg, L.; Keizer, K. I Am What I Am, by Looking Past the Present. Environ. Behav. 2014, 46, 626-657. [CrossRef] 